\title{
Atomic-Resolution Scanning Transmission Electron Microscopy with Segmented Annular All Field Detector
}

\author{
N. Shibata, ${ }^{1,2}$ S.D. Findlay ${ }^{3}$ and Y. Ikuhara ${ }^{1,4}$ \\ ${ }^{1}$ Institute of Engineering Innovation, The University of Tokyo, Tokyo 113-8656 Japan \\ ${ }^{2}$ PRESTO, Japan Science and Technology Agency, Saitama 332-0012, Japan \\ ${ }^{3}$ School of Physics, Monash University, Victoria 3800, Australia \\ ${ }^{4}$ Nanostructures Research Laboratory, Japan Fine Ceramic Center, Nagoya, 456-8587, Japan
}

Aberration-corrected scanning transmission electron microscopy (STEM) has become an indispensable tool for characterizing atomic-scale structure in materials and devices. In STEM, a finely focused electron probe is scanned across the specimen and transmitted and/or scattered electrons from a localized material volume are detected by the post specimen detector(s) as a function of raster position. By controlling the detector geometry, we have a lot of flexibility in determining the contrast characteristics of STEM images and the formation mechanisms involved. We have developed an area detector which we refer to as the "Segmented Annular All Field (SAAF)" detector and which is capable of atomic-resolution STEM imaging [1]. This area detector can obtain 16 simultaneous atomicresolution STEM images which are sensitive to the spatial distribution of scattered electrons on the detector plane [1]. The angle range can be easily controlled by changing camera length settings. In addition, we have a rotation capability for this detector, and thus we can precisely align the orientation of the detector relative to the crystallographic orientation of the specimen. The detector is easy to operate, and we have shown that the signals from individual segments can readily be combined to synthesize the signal corresponding to standard annular detectors, such as annular dark field. After this initial success, the second generation SAAF detector for the state-of-the art STEM is now being developed.

In this presentation, we will review recent and on-going findings from our exploration of new atomicresolution imaging modes using the SAAF detector. One possibility is atomic-resolution differential phase contrast (DPC) imaging [2]. It has been reported that, to a good approximation, DPC STEM images represent the gradient of the object potential (= fields) taken in the direction of the diagonally opposed detector segments, provided the object scatters weakly [3-5]. Here, we show atomic-resolution DPC STEM images of ferroelectric $\mathrm{BaTiO}_{3}$ observed from the [001] direction [2]. Fig. 1(a) shows the orientation relationship between the $\mathrm{BaTiO}_{3}$ crystal and the detector segments used. Fig. 1(b) shows the experimental difference image and its intensity profile projected over the vertical direction in the image. Whereas the DPC intensity profile of $(\mathrm{X}-\mathrm{Z})$ perpendicular to the polarization axis shows no asymmetric features, that of (W-Y) parallel to the polarization axis exhibits clear asymmetry as indicated by the arrows. Detailed theoretical analysis showed that the present profile asymmetry sensitively reflects the formation of electric dipoles in the unit cell [2]. Some application results of DPC STEM imaging for ferroelectics will be presented.

Another possibility is annular bright-field (ABF) imaging and its derivatives. Fig. 2(a) shows a schematic of the ABF detector geometry. We form "enhanced" (e)ABF images [6] by simply taking the difference between $\mathrm{ABF}$ images and the corresponding BF images using the SAAF detector. As shown in Fig. 2(b), we find that light element imaging can be selectively enhanced by this process. We 
anticipate that the SAAF detector will offer still further possibilities for new atomic-resolution STEM imaging modes useful for material characterization.

\section{References}

[1] N. Shibata et al., J. Electron Microscopy 59, 473 (2010).

[2] N. Shibata et al., Nature Phys., 8, 611 (2012).

[3] N.H. Dekkers and H. de Lang, Optik, 41, 452 (1974).

[4] H. Rose, Ultramicroscopy, 2, 251 (1977).

[5] W.C. Stewart, J. Opt. Soc. Am., 66, 813 (1976).

[6] S.D. Findlay et al., Ultramicroscopy, 136, 31 (2014).

[7] We thank Y. Kohno, H. Sawada and Y. Kondo (JEOL Ltd.) for their collaboration in the detector development. This work was supported by the PRESTO, JST and the JSPS KAKENHI Grant number 23686093. A part of this work was conducted in Research Hub for Advanced Nano Characterization, The University of Tokyo, under the support of "Nanotechnology Platform" (project No.12024046) by MEXT, Japan. This research was supported under the Discovery Projects funding scheme of the Australian Research Council (Project No. DP110101570).

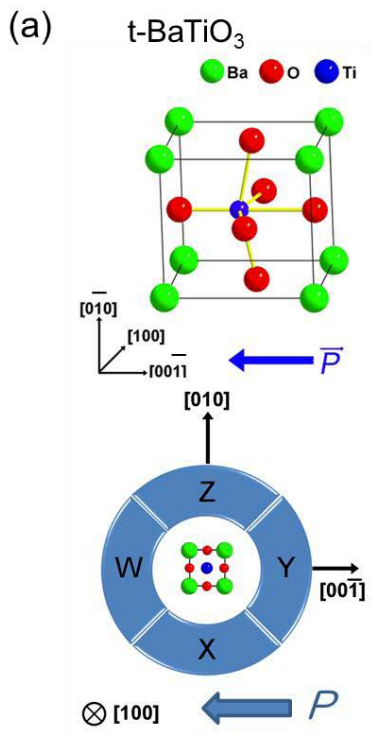

(b)
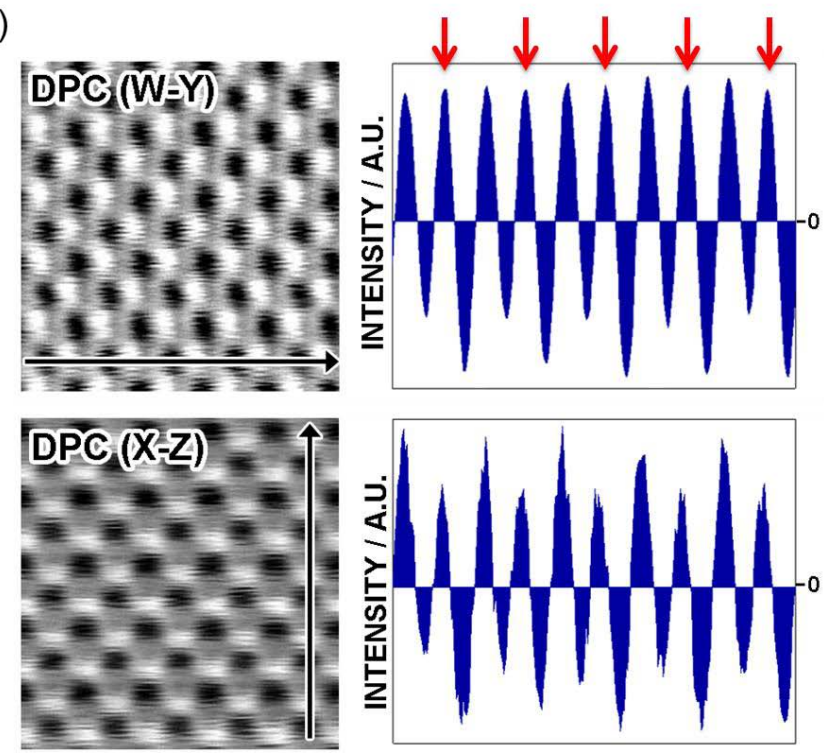

FIG. 1. Atomic-resolution DPC STEM of ferroelectric BaTiO3 [2].

(a)

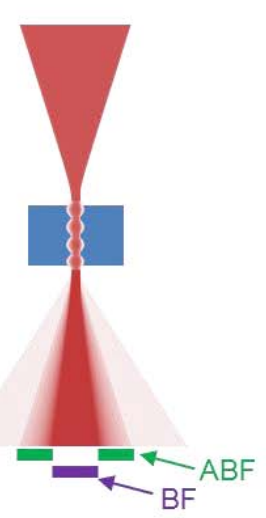

(b)

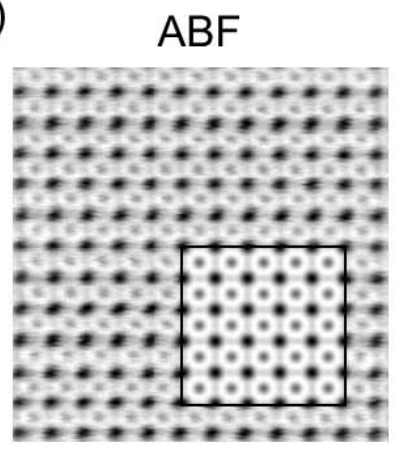

eABF

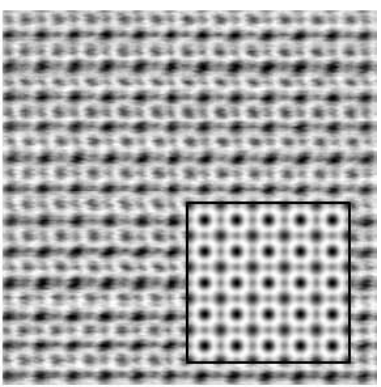

FIG. 2. ABF and eABF images of LaTiO3 [6]. 